\title{
Multidimensional Radiative Transfer in Accretion Environments
}

\author{
J. Steinacker, Th. Henning, and A. Menshchikov \\ $M P G$ Research Unit "Dust in Star-Forming Regions", Schillergäßchen \\ 2-3, 07745 Jena, Germany
}

\section{Introduction}

Most likely produced as thermal emission by dust grains, infrared emission from young stars and active galaxies reveals information about the internal energy source, the spatial distribution of the surrounding matter and its dynamical evolution. In this way, radiative transfer works as a bridge between HD/MHD accretion disk theory and observations. For young stellar objects and active galactic nuclei, the source geometry is of crucial importance for the modelling the emerging radiation. Observations indicate rotationally symmetric accretion disks, tori, or more complicated density structures around multiple sources, making multidimensional calculations necessary.

\section{Approximate 2D code}

With an efficient approximative $2 \mathrm{D}$ radiative transfer code, we have modelled the deeply embedded young stellar object L 1551-IRS 5 and derived its source structure (for details see Menshchikov \& Henning 1996). A thick flared disk model fits perfectly the broad-band photometry in the entire spectral range from visual to millimeter wavelengths. Intensity maps are in very good agreement with available linear scans and maps at $50 \mu \mathrm{m}, 100 \mu \mathrm{m}, 1.25 \mu \mathrm{m}$, and $1.3 \mathrm{~mm}$. Model visibilities fit very well the interferometry measurements at submm $/ \mathrm{mm}$ wavelengths $(870 \mu \mathrm{m}, 2.73 \mathrm{~mm})$ and confirm the presence of a compact and very dense core at the centre of IRS 5 . Model polarization maps at $1 \mu \mathrm{m}$ predicting both the polarization degree and overall pattern are in agreement with the observed ones. The thick flared disk model of IRS 5 with the opening angle of $90^{\circ}$ between the upper and lower conical surface can naturally account for the cross-shaped pattern recently observed at $730 \mu \mathrm{m}$.

Another target of recent modelling was HL Tau. Figure 1 shows an intensity map at $0.7 \mu \mathrm{m}$, convolved with a $0.5^{\prime \prime}$ beam. The viewing angle is $29.5^{\circ}$ above the midplane, the flared disk has an opening angle of $60^{\circ}$, the outer radius of the disk is $9600 \mathrm{AU}$, and the central object luminosity is $0.75 L_{\text {sun }}$.

\section{Full 3D code}

We present a new efficient method to solve the full 3D radiative transfer problem. The integro-differential transfer equation is cast into a large linear equation system using a second-order accurate finite differencing discretization, and by 


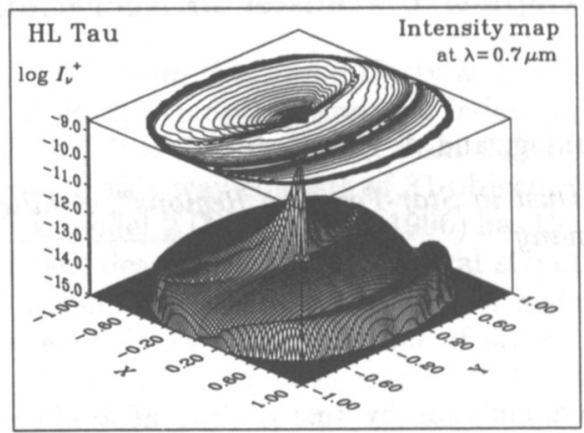

Figure 1. Intensity map of HL Tau at $\lambda=0.7 \mu \mathrm{m}$

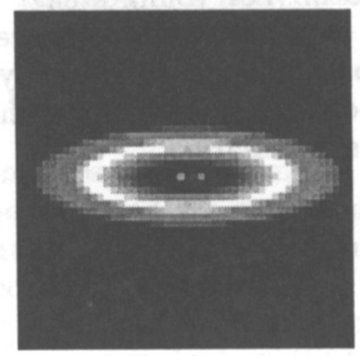

Figure 2. Intensity map for the inner part of a model binary system at $\lambda=1.65 \mu \mathrm{m}$

applying an optimized grid point distribution for the integration on the sphere (Steinacker, Maier, \& Thamm 1996). The equation system is solved using the fast and stable Bi-CGSTAB solver. This approach avoids any use of flux-limited approximations and allows for arbitrary scattering properties of the dust particles.

The self-consistent dust temperature is obtained by integrating the transfer equation over all frequencies and using the local energy balance equation to eliminate the planck function from the equation. This procedure requires large internal memory (about $1 \mathrm{~Gb}$ for resonable resolutions), but has the advantage of avoiding slowly converging $\Lambda$-iterations. Figure 2 shows the resulting intensity map for the inner part of a model binary system with a circumbinary accretion disk. Both the stars and the inner edge of the accretion disk are visible at $\lambda=1.65 \mu \mathrm{m}$.

\section{References}

Menshchikov, A., \& Henning, Th. 1996, A\&A, in press

Steinacker, J., Maier, U., \& Thamm, E. 1996, JQSRT, 56, 97 